\title{
Analysis of the Change of Artificial Intelligence to Online Consumption Patterns and Consumption Concepts
}

Longyue Bai ( $\sim$ bailongyuezh@163.com )

Jilin University

\section{Research Article}

Keywords: Artificial intelligence, online consumption, mobile payment, consumption concept

Posted Date: September 13th, 2021

DOl: https://doi.org/10.21203/rs.3.rs-851484/v1

License: (c) (1) This work is licensed under a Creative Commons Attribution 4.0 International License.

Read Full License

Version of Record: A version of this preprint was published at Soft Computing on January 8th, 2022. See the published version at https://doi.org/10.1007/s00500-021-06585-5. 


\title{
Analysis of the change of artificial intelligence to online consumption patterns and consumption concepts
}

\author{
Longyue Bai \\ College of the Humanities, Jilin University, Changchun, Jilin, 130012, China \\ Email: bailongyuezh@163.com
}

\begin{abstract}
In order to explore the change of artificial intelligence to online consumption patterns and consumption concepts, this paper explores the changes of artificial intelligence to online consumption patterns and consumption concepts, which are mainly realized through intelligent platforms.Moreover, this paper uses intelligent algorithms to improve the efficiency of system processing data, and uses data mining algorithms to process consumption patterns and consumption concept data.In addition, this paper studies the size and effect of the intermediary role played by payment mental accounts in payment costs and consumer purchase decisions, and studies the changes and effects of artificial intelligence on people's consumption patterns.Finally, this paper analyzes the changes of artificial intelligence to online consumption patterns and consumption concepts by investigating access methods and big data technology. Through investigation and research, we know that artificial intelligence can change consumption patterns, and make people more willing to accept this pattern of smart consumption, and it improves the convenience of people's consumption.
\end{abstract}

Keywords: Artificial intelligence; online consumption; mobile payment; consumption concept

\section{Introduction}

Artificial intelligence technology has not only changed people's lifestyles, but also changed people's payment methods. Therefore, research on artificial intelligence has a certain positive effect on the changes in online consumption patterns and consumption concepts.

In recent years, emerging mobile payments have developed rapidly, payment methods tend to be diversified and diversified, and payment scenarios have gradually expanded. Moreover, shopping forms are becoming more changeable, product origin is not restricted, and people are beginning to pursue diversity, individuality and taste. In addition, multiple life scenes are closely connected, consumer experience is gradually improved, consumer ideology changes, and shopping consumption time continues to shorten.It can be seen that emerging payment methods are changing people's daily consumption habits and reshaping people's lifestyles. In the new shopping environment, when consumers choose a specific payment method for payment, whether the consumer psychological feelings are the same, whether different psychological expectations will affect consumer product preferences and affect the final purchase decision is a question that needs to be studied [1].At present, scholars' research on the influence of different payment methods on purchasing decisions tends to focus on cash payment and bank card payment, while research on new payment methods is still relatively small.There is also a lack of research on the degree of preference of different types of products in payment methods, and there is no research with the help of EEG experiments [2]. Whether consumers pay the same attention to different types of products, whether different payment methods and product types cause consumers to have different emotions, whether different emotional changes affect consumers' product choices, and the cranial nerve mechanisms of consumers' purchase decision-making process still needs to be explored [3].

The impact of non-cash payment tools on residents' willingness to consume is mainly reflected in three aspects. First, non-cash payment tools provide residents with payment protection for consumption anytime and anywhere, and their convenience improves residents' propensity to consume and increase unexpected consumption. Second, the "mental account" effect of non-cash payment reduces the psychological loss of payment and directly increases residents' willingness to consume. Third, the interest-free credit overdraft function of non-cash payment instruments enhances residents' willingness to consume by enhancing the utility of consumption. Non-cash payment tools have the characteristics of convenience, safety, flexibility, etc., which avoids residents from restraining their impulse to consume due to insufficient cash, and there is no security concern about carrying cash for consumption. Residents can consume at any time. The payment tool with credit function can also enjoy credit support anytime and anywhere without any additional cost. Therefore, the convenience of non-cash payment tools stimulates the residents' surviving impulse to consume, enhances the willingness to consume, and accelerates the completion of consumption behavior. Due to the "mental account" effect of non-cash payment tools, it can give consumers a better consumer psychological experience than cash payment. 


\section{Related work}

Consumer purchasing decision refers to consumers choosing a purchasing plan that they think is the best under multiple selected plans in order to satisfy a certain demand [4]. This is the inner activity of the consumer before the actual purchase. The factors that affect consumers' purchasing decisions are very complex. From the perspective of consumers' own factors, income is an important variable that affects consumers' purchasing decisions, and relevant research has also achieved important results. Such as absolute income theory, relative income theory, life cycle theory and permanent income theory, etc., occupy a very important position in mainstream economics [5]. The development of anticipation theory in consumer purchase decision theory makes consumer decision research closer to reality. Literature [6] believes that income is uncertain, and residents will make reasonable adjustments between preventive demand and consumer purchasing decisions. Literature [7] believes that consumers' consumption expenditures generally depend on their lifetime total income, but they will fluctuate under the influence of uncertain factors. Literature [8] provides a more comprehensive explanation of the precautionary savings model. It is the caution and impatience of consumers that make them tend to maintain a fixed savings ratio. Payment methods have an impact on consumers' purchasing decisions. For example, payment methods that provide loans for consumption will stimulate consumer spending. This is explained from the perspective of liquidity. When consumers can improve current liquidity through credit and other channels, it can promote the increase in current consumption Literature [9] believes that compared with no liquidity constraints, the consumption demand under liquidity constraints is lower. According to the theory of liquidity constraints, the combination of low income and liquidity constraints will cause consumers to have "short-sighted behavior." Literature [10] analyzed this "short-sighted behavior" and pointed out that Chinese residents do not seek to maximize utility with a lifetime as a time span, but there are often specific spending peaks in different stages of their lives, such as house purchases and marriages. And children's education. At each stage, they must save to meet the corresponding spending peak and reduce consumption. With the establishment of the discipline of behavioral economics, some scholars have begun to study consumer behavior from the perspective of consumer psychology. This line of thinking makes the research on consumer behavior broader and closer to reality. Consumer's social status perception ability: The study of consumer behavior from the perspective of consumer psychology can be traced back to the "demonstration effect" [11]. Consumers' social status will affect consumers' specific consumption behaviors, but this method may Will make the preference set infinite; Literature [12] studies the characteristics of preferences from the perspective of psychology and experiment; Literature [13] believes that the environmental information around consumers (such as the consumption behavior of people around) will affect and benefit (in people The perception of unbiased circumstances) the consumer's consumer purchase decision. Consumer self-control ability: Literature [14] believes that consumers have both the impulse to consume and the ability to control themselves; Literature [15] believes that consumers' decision-making is a game between "impulsive short-term self" and "patient long-term self"; Literature [16] ] Through actual investigation, it is found that young people have relatively poor self-control ability and are more prone to impulsive consumption. In addition, the consumer's growth environment, educational background, and the composition of family members will all affect consumers' purchasing decisions.

With the application of information technology in the field of payment, electronic payment methods occupy an increasingly important position in the field of payment, and there is an increasing number of studies on electronic payment methods that stimulate consumer behavior. Literature [17] conducted an empirical study on the proportion of consumer expenditures and electronic payments in 50 countries around the world. The results revealed that there is a positive correlation between consumer expenditures and electronic payments. Electronic payments account for every proportion of consumer expenditures. Increased by $10 \%$, consumer spending increased by $0.5 \%$. Literature [18] believes that bank cards can stimulate the consumption demand of domestic residents, thereby stimulating economic growth. Literature [19] shows that a significant positive long-term equilibrium relationship has been established between total domestic consumption and bank card payment. The widespread use of bank cards is not only a substitute for cash payments, but also has an impact on economic growth. enhancement. Literature [20] believes that debit cards, as a typical representative of electronic payment methods, are of great significance for reducing cash flow and improving the efficiency of capital use.

\section{Data mining of consumption patterns and consumption concepts}

This article explores the changes of artificial intelligence to online consumption patterns and consumption concepts, which are mainly realized through intelligent platforms. Moreover, this paper uses intelligent algorithms to improve the efficiency of system data processing, and uses data mining algorithms to process consumption patterns and consumption concept data.

$$
\text { We are given an unlabeled training data set } X=\left\{x_{1}, x_{2}, \ldots, x_{p}\right\} \text {. The goal of the deep computing model based on crowdsourc- }
$$

ing is to select a subset $\left\{x_{1}, x_{2}, \ldots, x_{N}\right\}$ from a given training set and release it to crowdsourcing volunteers for labeling.After the marking is completed, the answers are collected and the correct rate of each crowdsourcing volunteer is evaluated based on the answers provided by the crowdsourcing volunteers, and the parameters of the in-depth calculation model are updated. The realization of a deep computing model based on crowdsourcing needs to solve the following two key issues:

(1) Crowdsourcing record selection. Crowdsourced record selection refers to selecting a suitable subset from the training data set as the crowdsourced release target, and allowing crowdsourced volunteers to provide tags.In practical applications, it is easy to obtain a large number of unlabeled samples, so it is impossible to release all unlabeled samples to crowdsourcing volunteers. On the one hand, not all unlabeled training data sets have an effect on parameter fine-tuning. On the other hand, labeling all unlabeled samples is time-consuming, laborious, and expensive.Therefore, only a subset can be selected for publishing. Crowdsourced record 
selection plays an important role in the fine-tuning of parameters and the training of classifiers. Choosing a suitable subset can significantly improve the effectiveness of fine-tuning the parameters of the depth calculation model.

The currently widely used method of crowdsourcing selection records is uncertainty sampling. Uncertainty sampling selects the sample with the largest uncertainty as the crowdsourced release object every time.If $p\left(z_{i}=1 \mid x_{i}\right)=\left(1+\exp \left(-\alpha^{T} x_{i}-\beta\right)\right)^{-1}$ is assumed to represent a two-class probability model, the uncertainty sampling selects the sample with the probability $p\left(z=1 \mid x_{i}\right)$ closest to $1 / 2$ as the crowdsourced release object.Therefore, for the binary classification problem, the uncertainty sampling can be expressed as the following optimization problem:

$$
\hat{x}=\min _{x}\left(\frac{1}{2}-p(z \mid x)\right)^{2}
$$

Generally speaking, uncertainty sampling is very effective for binary classification problems. However, uncertainty sampling is generally not suitable for multi-class models. At this time, a crowdsourced record selection algorithm based on the largest dilemma was proposed. The algorithm also selects the sample with the greatest uncertainty as the crowdsourced release object each time.If it is assumed that the probability that the sample $\mathrm{x}$ belongs to the class $\mathrm{z}$ is $\operatorname{Pr}\left(z_{i} \mid x_{i}, \theta\right)$, then the crowdsourced record selection algorithm based on the largest dilemma can be formalized as the optimization problem of formula (2):

$$
\hat{x}:=\max _{x}-\sum_{j=1}^{J} \operatorname{Pr}\left(z_{i} \mid x_{i}, \theta\right) \log \operatorname{Pr}\left(z_{i} \mid x_{i}, \theta\right)
$$

From formula (1) and formula (2), it can be seen that whether it is the method based on uncertainty sampling or the method of crowdsourced record selection based on the largest scale, it is necessary to establish an initial classifier in advance to determine the probability of each sample belonging to a specific class.Furthermore, the sample with the greatest uncertainty is selected as the crowdsourced release target. However, in most cases, the samples in the training set have no labels, and the initial classifier cannot be established. As a result, the above two methods are not applicable in the actual application process. Therefore, how to select crowdsourcing records has become the first key problem that needs to be solved to realize the deep computing model based on crowdsourcing.

(2) Answer integration. The goal of answer integration is to find the implicit true solution from the answers provided by a large number of crowdsourced volunteers. We assume that the unlabeled sample $\mathrm{x}$ is labeled by $\mathrm{T}$ volunteers, and the answer set is $\left(x_{i}^{(1)}, x_{i}^{(12)}, \ldots, x_{i}^{(T)}\right)$ The purpose of answer integration is to find the true label of the sample $x_{i}$ from the set $\left(x_{i}^{(1)}, x_{i}^{(12)}, \ldots, x_{i}^{(T)}\right)$ lenges to effective answer integration strategies. On the one hand, crowdsourcing volunteers have different professional levels and different knowledge backgrounds, which may lead to conflicts or uncertainties in the answers provided by crowdsourcing volunteers. On the other hand, the difficulty of the questions is different, and even the same volunteer, the correctness of the answers given to different questions is not the same.It can be seen that the correctness of answers given by volunteers depends on two elements: the professional level and knowledge background of the volunteers, and the field and difficulty of the problem.In response to the problem of crowdsourcing technology answer integration, Raykar et al. proposed a SLME algorithm in 2010, which is currently recognized as one of the most effective algorithms. The algorithm uses two indicators in statistics-sensitivity and specificity instead of the confusion matrix to measure the correctness of each volunteer's answer.However, this method can only be used for binary classification problems, not for multi-classification problems. Therefore, how to design an effective answer integration method and find the implicit correct label from the answers provided by volunteers is the second challenge that needs to be solved to realize the deep computing model based on crowdsourcing.

In recent years, researchers have proposed a variety of algorithms to solve the problem of answer integration in crowdsourcing technology. These algorithms can be roughly divided into two categories: non-iterative algorithms (such as majority voting algorithm, Honeypot algorithm, ELICE algorithm, etc.) and iterative algorithms (such as expectation maximization algorithm, GLAD algorithm, SLME algorithm, and ITER algorithm, etc.).

We assume that the crowdsourced sample set $O=\left\{o_{1}, o_{2}, \ldots, o_{n}\right\}$ contains n samples, and the volunteer set is $T=\left\{t_{1}, t_{2}, \ldots, t_{k}\right\}$, that is, each sample is labeled by $\mathrm{k}$ volunteers, and there are m possible labels $L=\left\{l_{1}, l_{2}, \ldots, l_{m}\right\}$ (that is, m classes) in total. $a_{i j}$ represents the label that volunteer $t_{j}$ gave to sample ${ }^{o_{i}}$.Then, the label matrix M labeled by all volunteers for all samples can be obtained as shown in formula (3)[21]:

$$
M=\left(\begin{array}{ccc}
a_{11} & \cdots & a_{1 k} \\
\vdots & \ddots & \vdots \\
a_{n 1} & \cdots & a_{n k}
\end{array}\right)
$$

Generally speaking, the answer integration algorithm takes the matrix $M$ as input, and the integrated value set $\gamma=\left\{\gamma_{1}, \gamma_{2}, \ldots, \gamma_{n}\right\}$ is solved, where $\gamma_{i} \in L$ represents the integrated label of the sample ${ }^{o_{i}}$.We assume that $\operatorname{Pr}\left\{X_{i}=l_{z}\right\}$ 
represents the probability that the object ${ }^{o_{i}}$ belongs to the class $l_{z}$, and the answer integration algorithm uses different models to estimate $\operatorname{Pr}\left\{X_{i}=l_{z}\right\}$, and then calculates the integrated label of the sample ${ }^{o_{i}}$ by formula (4):

$$
\gamma_{i}=\arg \max _{l_{z} \in L} \operatorname{Pr}\left\{X_{i}=l_{z}\right\}
$$

The non-iterative algorithm uses a heuristic strategy to calculate the integrated value of each problem. The most typical noniterative algorithm is the majority voting method.We assume that the crowdsourced volunteers give $\mathrm{k}$ answers for sample ${ }_{i}$. The majority voting method first counts the number of answers given by volunteers that contain the label $l_{z}$, and further calculates the probability that the sample ${ }^{O_{i}}$ belongs to the class $l_{z}$ as shown in formula (5):

$$
\operatorname{Pr}\left\{X_{i}=l_{z}\right\}=\left.\frac{1}{k} \sum_{j=1}^{k} 1\right|_{a_{i j}=l_{z}}
$$

The majority voting algorithm is simple and easy to implement. However, the algorithm does not consider the impact of each volunteer's professional level and knowledge background on the answers given. Therefore, in actual applications, the effect is not ideal.

This paper proposes a crowdsourced record selection algorithm based on fuzzy clustering and maximum quietness. Fuzzy clustering algorithm can accurately describe the uncertain relationship between patterns, and is widely used in many fields such as image processing, pattern recognition, and computer vision. Fuzzy clustering assigns each data sample to different classes according to the degree of membership, and the sum of the membership degrees of each class to which the sample belongs is 1.The entire data object set is $X=\left\{x_{1}, x_{2}, \ldots, x_{n}\right\}$, and each object has $m$ attributes $\left\{a_{1}, a_{2}, \ldots, a_{m}\right\}$, that is, $A=\left\{a_{1}, a_{2}, \ldots, a_{m}\right\}$ is the attribute set. $x_{i j}$ represents the $\mathrm{j}$-th attribute value of the $\mathrm{i}$-th object.Fuzzy clustering is defined as a cxn membership matrix $U=\left\{u_{i j}\right\}$, where $u_{i j}$ represents the membership of the $\mathrm{j}$-th object belonging to the $\mathrm{i}$-th class, $\mathrm{n}$ represents the number of data objects, and $\mathrm{c}$ represents the clustering data.The formal definition of fuzzy clustering algorithm is shown in formula (6):

$$
M_{f c n}=\left\{U \in R^{c \times n} \mid u_{i j} \in[0,1] \forall j, i 0<\sum_{j=1}^{n} u_{i j}<n, \forall i ; \sum_{j=1}^{c} u_{i j}=1, \forall j\right\}
$$

We assume that the cluster center of fuzzy clustering is $V=\left\{v_{1}, v_{2}, \ldots, v_{c}\right\}$, and the objective function of fuzzy clustering is:

$$
J_{m}(U, V)=\sum_{i=1}^{c} \sum_{k=1}^{n} u_{i j}^{m}\left\|x_{k}-v_{i}\right\|^{2}
$$

In order to minimize the objective function, the updated membership degree and cluster center formulas of the fuzzy clustering algorithm are shown in (8) and (9):

$$
\begin{gathered}
u_{i j}=\left[\sum_{j=1}^{c}\left(\frac{\left\|x_{k}-v_{i}\right\|}{\left\|x_{k}-v_{j}\right\|}\right)^{\frac{2}{m-1}}\right]^{-1} \\
v_{i}=\sum_{k=1}^{n} u_{i k}^{m} x_{k} / \sum_{k=1}^{n} u_{i k}^{m}
\end{gathered}
$$

The initial depth calculation model obtained by pre-training is used to learn the features of each sample instance, and the feature tensor is expanded into the corresponding vector form to obtain the training subset $\tilde{X}=\left\{x_{1}, x_{2}, \ldots, x_{n}\right\}$ represented by the feature vector.We assume that the number of categories is c, the corresponding clustering center is $V=\left\{v_{1}, v_{2}, \ldots, v_{c}\right\}$, and use fuzzy clustering algorithm to cluster $\tilde{X}=\left\{x_{1}, x_{2}, \ldots, x_{Q}\right\}$.

After the clustering is completed, the clustering result of the data subset $\tilde{X}=\left\{x_{1}, x_{2}, \ldots, x_{Q}\right\}$ $U=\left\{u_{i j}\right\}(i=1,2, \ldots, c ; j=1,2, \ldots, \mathrm{Q})$ class.Next, this paper uses information entropy $\operatorname{En}\left(x_{i}\right)$ to measure the uncertainty of each sample, as shown in formula (10):

$$
E n\left(x_{i}\right)=-\sum_{j=1}^{c} u_{j i} \log u_{j i}
$$

The $\mathrm{N}$ data samples with the largest information entropy are selected as crowdsourced publishing objects. From the main process of the algorithm, it can be seen that the main step of the algorithm is to perform fuzzy clustering on the samples, and the time 
complexity of updating the fuzzy matrix and the clustering center is $O(Q \times c)$.Therefore, the overall time complexity of the entire algorithm is approximately $O(Q \times c \times k)$, where k is the number of iterations.

The SLME algorithm is currently recognized as the most effective answer integration algorithm, but this algorithm is only suitable for two classification problems. In response to this problem, this paper uses the expectation maximization algorithm to extend the SLME algorithm by introducing a confusion matrix to integrate crowdsourced answers to multi-classification problems.In the process of answer integration, the professional level of each volunteer is evaluated at the same time and the parameters of the in-depth calculation model are fine-tuned. This section elaborates on the implementation process of the answer integration algorithm proposed in this paper.

We assume that the data set $X=\left\{x_{1}, x_{2}, \ldots, x_{N}\right\}$ is a set of crowdsourced records selected from the unlabeled training data set, and each data sample is annotated by $\mathrm{T}$ volunteers. ${ }^{y_{i}^{(t)}}$ represents the label provided by the t-th volunteer for sample ${ }^{x_{i}}$.We assume that the number of categories is $\mathrm{J}$, and ${ }^{z_{i}}$ represents the true unknown label of sample $x_{i}$. The confusion matrix is $\left\{\pi_{i j}^{(t)}\right\}$, and $\pi_{i j}^{(t)}$ represents the probability that volunteer $\mathrm{t}$ will assign an object in class $\mathrm{i}$ to class $\mathrm{j}$.Therefore, the goal of the answer integration algorithm designed in this paper is to use the expectation maximization algorithm to estimate the parameter set $\left\{w, \pi_{i j}^{(t)}\right\}$, where w represents the parameter set of the depth calculation model.

We assume that the annotations of the crowdsourcing records by the crowdsourcing volunteers are independent of each other, and the answers provided only depend on the true labels of the samples.The answer provided by the crowdsourcing volunteers is $Y=\left\{x_{i} ; \mathrm{y}_{i}^{(1)}, \mathrm{y}_{i}^{(2)}, \ldots, \mathrm{y}_{i}^{(T)}\right\}_{i=1}^{N}$, and the likelihood function of the parameter $\theta=\left\{w, \pi_{i j}^{(t)}\right\}$ is defined as shown in formula (11):

$$
\operatorname{Pr}(Y \mid \theta)=\prod_{i=1}^{N} \operatorname{Pr}\left\{\mathrm{y}_{i}^{(1)}, \mathrm{y}_{i}^{(2)}, \ldots, \mathrm{y}_{i}^{(T)} \mid x_{i} ; \theta\right\}
$$

By introducing the implicit true label $Z=\left\{z_{1}, z_{2}, \ldots, z_{N}\right\}$, the likelihood function (11) can be decomposed into formula (12):

$$
\begin{aligned}
& \operatorname{Pr}(Y \mid \theta)=\prod_{i=1}^{N} \operatorname{Pr}\left\{\mathrm{y}_{i}^{(i)}, \mathrm{y}_{i}^{(2)}, \ldots, \mathrm{y}_{i}^{(T)} \mid x_{i} \theta \theta\right\}=\prod_{i=1}^{N} \sum_{i=1} \operatorname{Pr}\left\{\mathrm{y}_{i}^{(1)}, \mathrm{y}_{i}^{(2)}, \ldots, \mathrm{y}_{i}^{(T)}, z_{i} \mid x_{i}, \theta\right\} \\
& =\prod_{i=1}^{N} \sum_{i=1} \operatorname{Pr}\left\{\mathrm{y}_{i}^{(1)}, \mathrm{y}_{i}^{(2)}, \ldots, \mathrm{y}_{i}^{(T)}, z_{i} \mid x_{i} ; \theta\right\} \operatorname{Pr}\left(z_{i} \mid x_{i}, \theta\right)
\end{aligned}
$$

When the implicit real label $\mathrm{z}$ is given, and each volunteer independently annotates the crowdsourced record, the formula (13) can be obtained:

$$
\begin{aligned}
& \operatorname{Pr}\left\{\mathrm{y}_{i}^{(1)}, \mathrm{y}_{i}^{(2)}, \ldots, \mathrm{y}_{i}^{(T)} \mid z_{i} ; \theta\right\}=\prod_{i=1}^{N} \operatorname{Pr}(y)=\prod_{i=1}^{N} \sum_{i=1} \operatorname{Pr}\left\{\mathrm{y}_{i}^{(1)}, \mathrm{y}_{i}^{(2)}, \ldots, \mathrm{y}_{i}^{(T)}, \mathrm{z}_{i} \mid x_{i} ; \theta\right\} \\
& =\prod_{i=1}^{N} \sum_{z_{i}} \operatorname{Pr}\left\{\mathbf{y}_{i}^{(1)}, \mathrm{y}_{i}^{(2)}, \ldots, \mathrm{y}_{i}^{(T)}, \mathbf{z}_{i} \mid x_{i} ; \theta\right\} \operatorname{Pr}\left(z_{i} \mid x_{i} ; \theta\right)
\end{aligned}
$$

Amongthem,

$$
\sigma\left(y_{i}^{(t)}, j\right)=\left\{\begin{array}{l}
1 \text { if } y_{i}^{(t)}=j \\
0 \text { otherwise }
\end{array}\right.
$$

$\operatorname{Pr}\left\{z_{i} \mid x_{i} ; \theta\right\}$

represents the output of the depth calculation model. For the convenience of explanation, in the next part, we set $\mathrm{P}_{i}=\operatorname{Pr}\left\{z_{i} \mid x_{i} ; \theta\right\}$.Therefore, the parameter $\theta=\left\{w, \pi_{i j}^{(t)}\right\}$ can be estimated by the following optimization problem:

$$
\theta:=\arg \max _{\theta}\{\operatorname{Pr}(Y \mid \theta)\}
$$

By taking the logarithm of formula (15), it is transformed into the optimization problem shown in (16):

$$
\theta:=\arg \max _{\theta}\{\ln \operatorname{Pr}(Y, Z \mid \theta)\}=\sum \ln \sum \operatorname{Pr}\left(y_{i}^{l}, \cdots y_{i}^{(T)} \mid Z_{i} ; \theta\right) \cdot \operatorname{Pr}\left(Z_{i} \mid x_{i} ; \theta\right)
$$

This paper uses the expectation maximization algorithm to solve the optimization problem shown in formula (15) through the following steps.

(1) Initialization

This paper uses the majority voting method to initialize the integrated value ${ }^{\mu_{i}}$ according to the crowdsourcing matrix $Y=\left\{x_{i} ; \mathrm{y}_{i}^{(1)}, \mathrm{y}_{i}^{(2)}, \ldots, \mathrm{y}_{i}^{(T)}\right\}_{i=1}^{N}$, and uses $\mu_{i}$ to initialize the confusion matrix $\left\{\pi_{i j}^{(t)}\right\}$.

(2) E-Step 
According to the estimated value of the crowdsourcing matrix $Y=\left\{x_{i} ; \mathrm{y}_{i}^{(1)}, \mathrm{y}_{i}^{(2)}, \ldots, \mathrm{y}_{i}^{(T)}\right\}_{i=1}^{N}$ and the current parameter $\theta=\left\{w, \pi_{i j}^{(t)}\right\}$, the conditional expectation is shown in formula (17):

$$
E\{\ln \operatorname{Pr}(Y, Z \mid \theta)\}=\sum_{i=1}^{N} \sum_{z_{i}} u_{i} \ln a_{i} p_{i}
$$

Among them, $a_{i}=\operatorname{Pr}\left\{\mathrm{y}_{i}^{(1)}, \mathrm{y}_{i}^{(2)}, \ldots, \mathrm{y}_{i}^{(T)} \mid z_{i} ; \theta\right\}$

According to Bayes' theorem, the calculation formula is shown in formula (18):

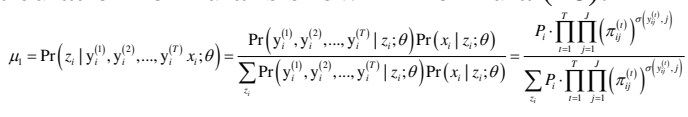

(3)M-Step

According to the estimated value of the current integrated value $\mathrm{u}$, the formula for calculating the confusion matrix $\left\{\pi_{i j}^{(t)}\right\}$ is shown in formula (19):

$$
\left\{\pi_{i j}^{(t)}\right\}=\frac{\sum_{i=1}^{N} \mu_{i} \cdot \sigma\left(y_{i}^{(t)}, j\right)}{\sum_{i=1}^{N} \mu_{i}}
$$

The weight $w$ is updated by the high-order backpropagation algorithm of the depth calculation model.

In each iteration of the answer integration algorithm, E-Step and M-Step are calculated through the forward propagation and back propagation processes of the deep calculation model, respectively. E-Stcp and M-Step are repeated to update the depth calculation model until convergence.

\section{Analysis of the changes of artificial intelligence to online consumption patterns and consumption concepts}

Artificial intelligence mainly changes people's lifestyles and consumption patterns. This article focuses on the study of artificial intelligence on the changes in people's consumption patterns and its impact.

The payment method affects the consumer's purchasing decision. The reason why payment methods affect consumers' purchasing decisions is that when consumers make economic decisions, they will measure the cost-effectiveness of the behavior according to the law of cost-benefit. When the cost of the behavior exceeds the benefits, the behavior is considered feasible.In the payment process, consumers will inevitably encounter various costs, such as one or several of time cost, holding cost, identification cost, and switching cost.Consumers will make mental accounting calculations of payment costs based on their sensitivity to different payment cost components, and make a purchase decision of buying or not, buying more and buying less. Figure 1 shows a preliminary model of the influence process of payment methods on consumers' purchasing decisions:

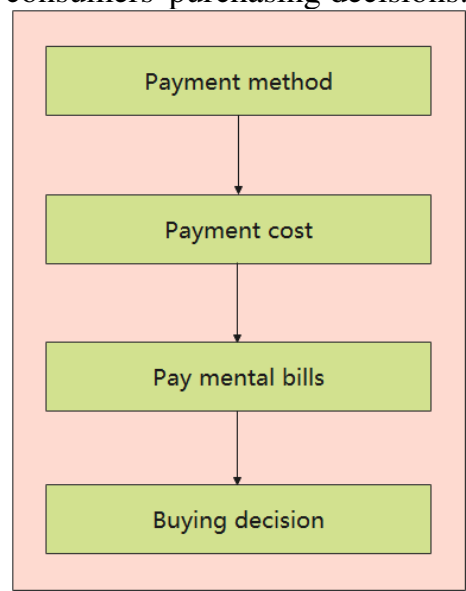

Figure 1 Preliminary model of the influence mechanism of payment methods on consumers' purchasing decisions

Different payment methods have different payment costs, resulting in a more complicated situation of different payment mental accounts. The influence process of payment methods on consumers' purchasing decisions is shown in Figure 2. 


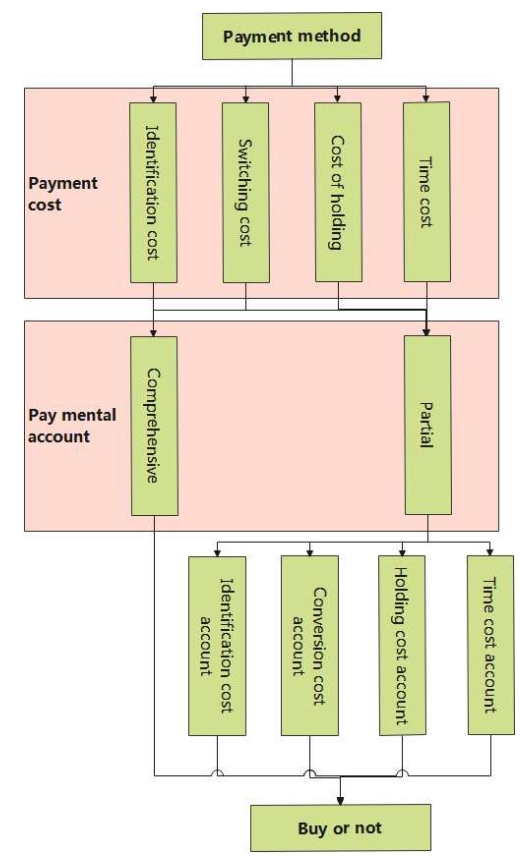

Figure 2 The influence process of payment methods on consumers' purchasing decisions The changes in the consumer's purchase decision-making process under the influence of payment methods are shown in Figure 3:
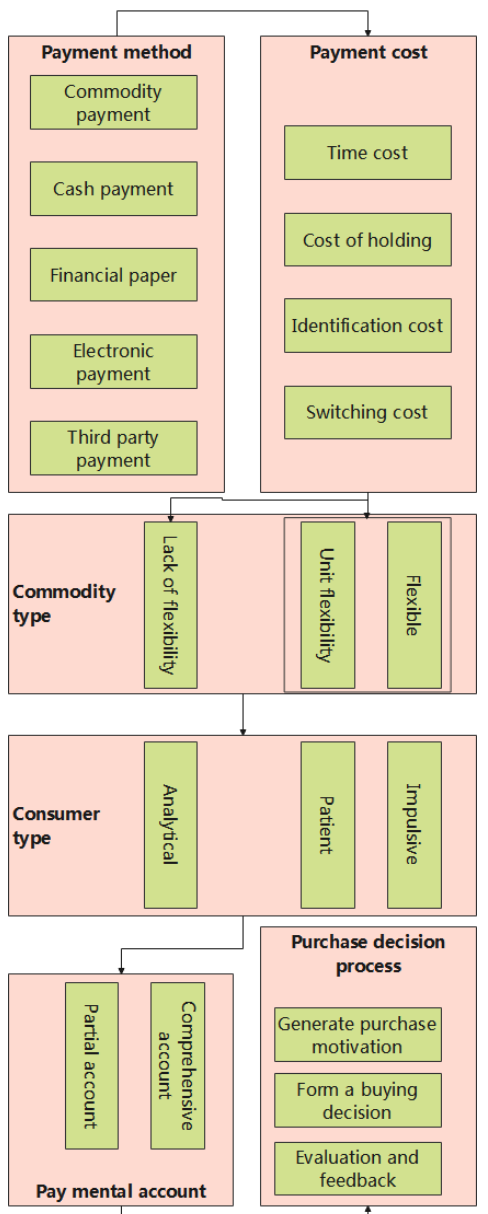

Figure 3 The influence mechanism of payment methods on consumers' purchasing decisions

This article studies the size and effect of the intermediary role played by payment mental accounts in payment costs and consumer purchase decisions. The specific research model is shown in Figure 4. 


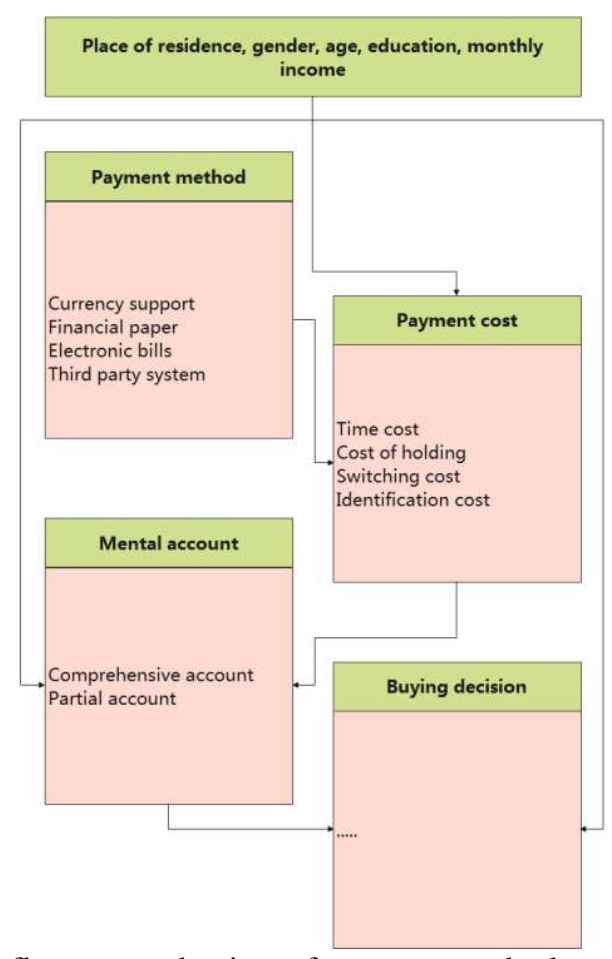

Figure 4 Model diagram of the influence mechanism of payment methods on consumers' purchasing decisions

This article analyzes the changes of artificial intelligence to online consumption patterns and consumption concepts through survey interviews and big data technology, obtains a large amount of data through questionnaires, and counts the changes of artificial intelligence to people's consumption patterns and consumption concepts. The research on the transformation of artificial intelligence on consumption patterns is obtained through data statistics. This article mainly counts payment method evaluation, acceptance, convenience, and prospect evaluation of artificial intelligence payment methods. The results are shown in Table 1 to Table 4 and Figure 5 to Figure 8.

Table 1 Evaluation of payment methods

\begin{tabular}{|c|c|c|c|c|c|}
\hline Number & Payment evaluation & Number & Payment evaluation & Number & Payment evaluation \\
\hline 1 & 67.50 & 11 & 93.44 & 21 & 22 \\
\hline 2 & 87.10 & 12 & 68.77 & 23 & 91.54 \\
\hline 3 & 78.27 & 13 & 90.16 & 24 & 83.93 \\
\hline 4 & 90.53 & 14 & 81.93 & 25 & 92.70 \\
\hline 5 & 94.68 & 15 & 67.43 & 26 & 88.45 \\
\hline 6 & 87.93 & 16 & 77.97 & 27 & 82.50 \\
\hline 7 & 90.45 & 17 & 82.04 & 28 & 70.56 \\
\hline 8 & 93.24 & 18 & 82.73 & 29 & 73.77 \\
\hline 9 & 92.91 & 19 & 72.22 & & 79.24 \\
\hline 10 & 86.43 & 20 & 85.84 & & 30 \\
\hline
\end{tabular}

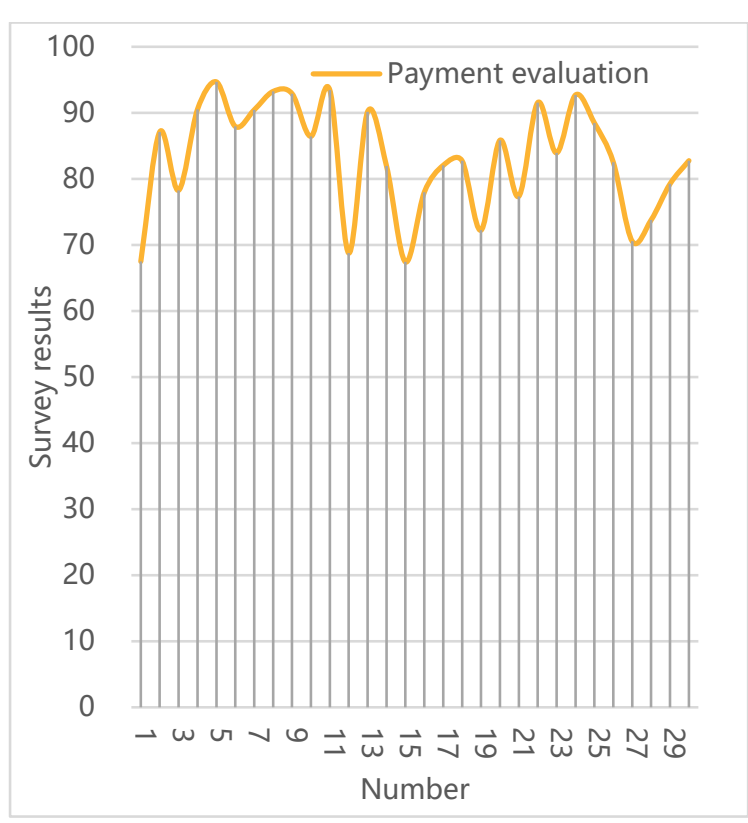


Figure 5 Statistical results of payment method evaluation

Table 2 Acceptance

\begin{tabular}{|c|c|c|c|c|c|}
\hline Number & Acceptance & Number & Acceptance & Number & Acceptance \\
\hline 1 & 80.05 & 11 & 70.17 & 21 & 60.64 \\
\hline 2 & 71.03 & 12 & 88.28 & 22 & 72.41 \\
\hline 3 & 61.93 & 13 & 85.68 & 23 & 63.88 \\
\hline 4 & 89.97 & 14 & 65.15 & 24 & 81.02 \\
\hline 5 & 65.18 & 15 & 82.65 & 25 & 67.35 \\
\hline 6 & 72.27 & 16 & 64.54 & 26 & 89.24 \\
\hline 7 & 64.07 & 17 & 90.43 & 27 & 77.67 \\
\hline 8 & 84.55 & 18 & 88.08 & 28 & 82.92 \\
\hline 9 & 65.83 & 19 & 90.77 & 29 & 59.89 \\
\hline 10 & 73.33 & 20 & 68.39 & 30 & 77.64 \\
\hline
\end{tabular}

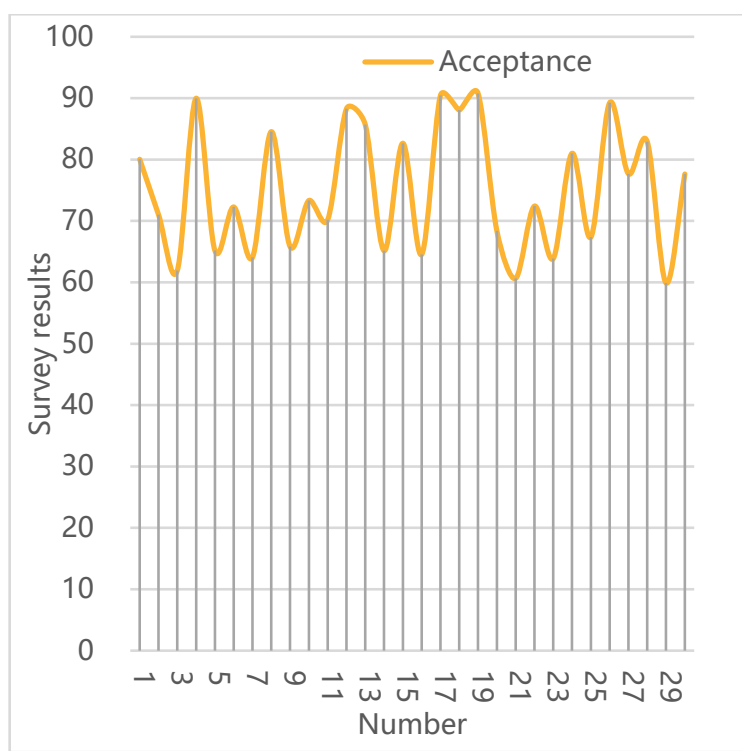

Figure 6 Statistical results of acceptance

Table 3 Convenience

\begin{tabular}{|c|c|c|c|c|c|}
\hline Number & Convenience & Number & Convenience & Number & Convenience \\
\hline 1 & 76.09 & 11 & 86.12 & 21 & 74.33 \\
\hline 2 & 83.30 & 12 & 84.98 & 22 & 83.21 \\
\hline 3 & 96.62 & 13 & 89.00 & 23 & 84.63 \\
\hline 4 & 88.48 & 14 & 86.00 & 24 & 97.22 \\
\hline 5 & 85.86 & 15 & 90.89 & 25 & 77.70 \\
\hline 6 & 82.09 & 16 & 95.16 & 26 & 75.18 \\
\hline 7 & 98.78 & 17 & 93.26 & 28 & 96.13 \\
\hline 8 & 76.67 & 18 & 92.98 & 29 & \\
\hline 10 & 95.14 & 19 & 82.23 & 30 & \\
\hline
\end{tabular}




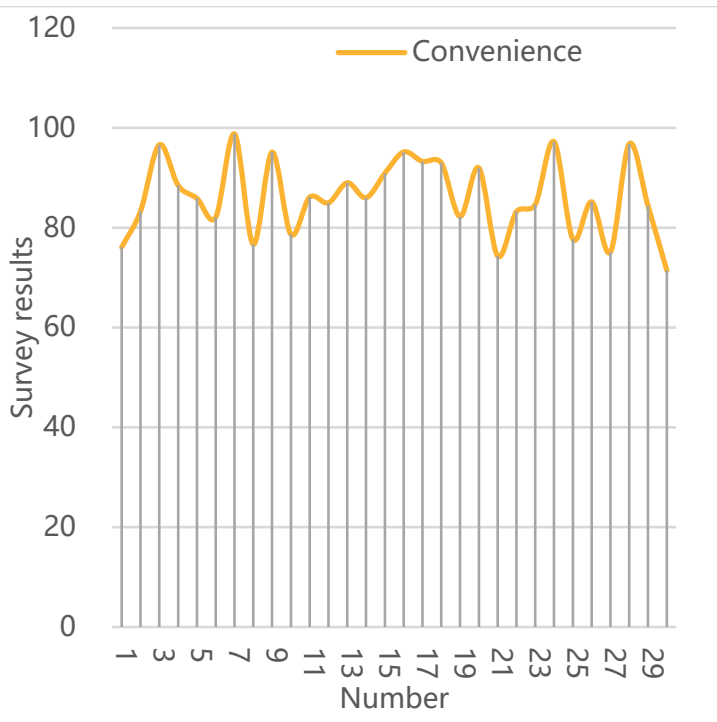

Figure 7 Statistical results of convenience

Table 4 Prospect evaluation

\begin{tabular}{|c|c|c|c|c|c|}
\hline Number & Prospect evaluation & Number & Prospect evaluation & Number & Prospect evaluation \\
\hline 1 & 85.14 & 11 & 91.12 & 21 & 93.96 \\
\hline 2 & 96.24 & 12 & 85.80 & 22 & 97.61 \\
\hline 3 & 97.71 & 13 & 84.49 & 23 & 91.81 \\
\hline 4 & 84.33 & 14 & 92.23 & 24 & 89.81 \\
\hline 5 & 98.04 & 15 & 88.61 & 25 & 94.81 \\
\hline 6 & 95.49 & 16 & 88.88 & 26 & 92.13 \\
\hline 7 & 94.95 & 17 & 89.41 & 27 & 98.66 \\
\hline 8 & 94.45 & 18 & 95.04 & 28 & 90.30 \\
\hline 9 & 88.91 & 19 & 85.29 & 29 & 91.37 \\
\hline 10 & 93.64 & 20 & 84.18 & 30 & 97.80 \\
\hline
\end{tabular}

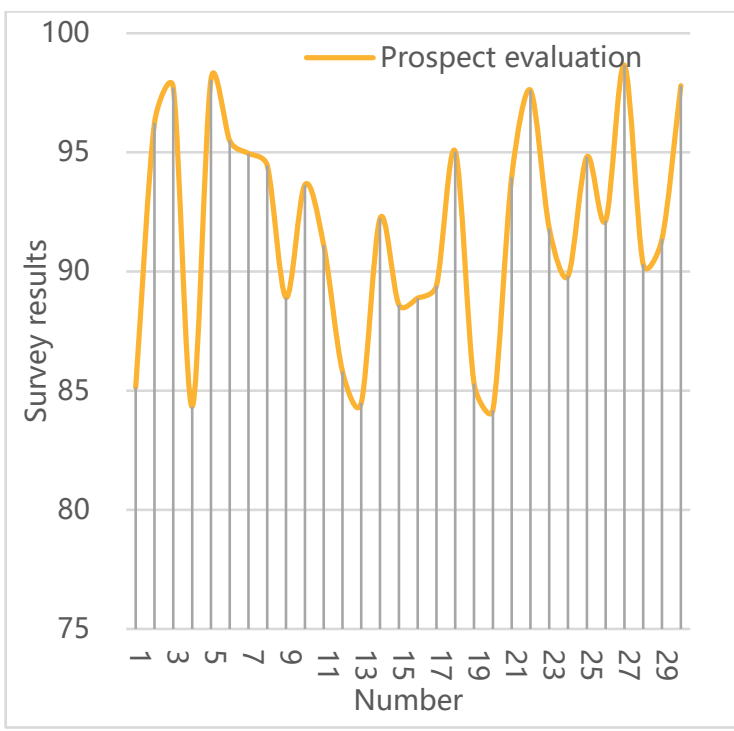

Figure 8 Statistical results of prospect evaluation

From the above research results, the intelligent evaluation model constructed in this paper can effectively evaluate the changes in artificial intelligence network consumption patterns and consumption concepts. At the same time, it can be seen from the above analysis that artificial intelligence can change the way people consume, and people are more willing to accept this way of smart consumption, and it improves the convenience of people's consumption.

\section{Conclusion}

When consumers make purchase decisions, the feeling of pity that comes from paying with cash, that is, the loss of mental accounts, can be diluted by artificial intelligence payments. The reason is that the mental account effect of artificial intelligence payment can reduce the price that residents pay for consumption, that is, the reduction of personal fund balance, and the intuitive 
feeling of this process is much weaker. Therefore, artificial intelligence payment tools will make it easier to make people want to buy, and the willingness to consume will be greatly enhanced, which will allow consumers to buy quickly or over-purchase.

The interest-free credit overdraft function of the artificial intelligence payment tool separates the actual consumption day of the residents from the fund payment day. Moreover, this time interval is an interest-free repayment period, and through this interval, consumers can obtain the interest utility brought about by the time difference of funds. This part of the utility is obtained in addition to cash consumption. In other words, the interest-free credit overdraft function of the artificial intelligence payment tool allows consumers to obtain more utility.In addition, card issuing banks or third-party payment institutions through marketing activities with merchants allow consumers to spend less money to enjoy the same goods or services consumption, which directly stimulates consumer desire. Therefore, artificial intelligence payment tools increase residents' willingness to consume by improving consumption utility.

\section{Declarations}

\section{Funding}

No funding is received.

Research involving Human Participants and/or Animals:

Not applicable

Informed consent:

Not applicable

\section{Conflicts of interests}

The authors declare that they have no conflicts of interest.

\section{Availability of data and material}

The data in this paper include calculation result data and Finite element analysis data. All the data used to support the findings of this study are included within the article. There are not any restrictions on data access.

Code availability

Not Applicable.

\section{Authors' Contributions}

Longyue Bai have participated in the designing framework, collecting data, analysis and interpretation of data, drafting, reviewing and approving the manuscript.

\section{References}

[1] Bulut Z A, KökalanÇımrin F, Doğan O. Gender, generation and sustainable consumption: Exploring the behaviour of consumers from Izmir, Turkey[J]. International Journal of Consumer Studies, 2017, 41(6): 597-604.

[2] Van Zanten H H E, Herrero M, Van Hal O, et al. Defining a land boundary for sustainable livestock consumption[J]. Global change biology, 2018, 24(9): 4185-4194.

[3] Zwanka R J, Buff C. COVID-19 generation: A conceptual framework of the consumer behavioral shifts to be caused by the COVID-19 pandemic[J]. Journal of International Consumer Marketing, 2021, 33(1): 58-67.

[4] Liu Y, Qu Y, Lei Z, et al. Understanding the evolution of sustainable consumption research[J]. Sustainable Development, 2017, 25(5): 414-430.

[5] Ali B. Impact of COVID-19 on consumer buying behavior toward online shopping in Iraq[J]. Ali, BJ (2020). Impact of COVID-19 on consumer buying behavior toward online shopping in Iraq. Economic Studies Journal, 2020, 18(42): 267280.

[6] Liu Y, Qu Y, Lei Z, et al. Understanding the evolution of sustainable consumption research[J]. Sustainable Development, 2017, 25(5): 414-430.

[7] Ali B. Impact of COVID-19 on consumer buying behavior toward online shopping in Iraq[J]. Ali, BJ (2020). Impact of COVID-19 on consumer buying behavior toward online shopping in Iraq. Economic Studies Journal, 2020, 18(42): 267280 .

[8] Schmidt A L, Zollo F, Del Vicario M, et al. Anatomy of news consumption on Facebook[J]. Proceedings of the National Academy of Sciences, 2017, 114(12): 3035-3039.

[9] Alfredsson E, Bengtsson M, Brown H S, et al. Why achieving the Paris Agreement requires reduced overall consumption and production[J]. Sustainability: Science, Practice and Policy, 2018, 14(1): 1-5.

[10] Ness D A, Xing K. Toward a Resource - Efficient Built Environment: A Literature Review and Conceptual Model[J]. Journal of Industrial Ecology, 2017, 21(3): 572-592.

[11] Bruel A, Kronenberg J, Troussier N, et al. Linking industrial ecology and ecological economics: A theoretical and empirical foundation for the circular economy[J]. Journal of Industrial Ecology, 2019, 23(1): 12-21.

[12] Lin S T, Niu H J. Green consumption: E nvironmental knowledge, environmental consciousness, social norms, and purchasing behavior[J]. Business Strategy and the Environment, 2018, 27(8): 1679-1688.

[13] Evans D M. What is consumption, where has it been going, and does it still matter?[J]. The Sociological Review, 2019, 67(3): 499-517.

[14] Dwyer T, Martin F. Sharing news online: Social media news analytics and their implications for media pluralism 
policies[J]. Digital journalism, 2017, 5(8): 1080-1100.

[15] Iqbal S, Bilal A R, Nurunnabi M, et al. It is time to control the worst: testing COVID-19 outbreak, energy consumption and CO 2 emission[J]. Environmental Science and Pollution Research, 2021, 28(15): 19008-19020.

[16] Venter C, Greenhawt M, Meyer R W, et al. EAACI position paper on diet diversity in pregnancy, infancy and childhood: Novel concepts and implications for studies in allergy and asthma[J]. Allergy, 2020, 75(3): 497-523.

[17] Nelson J L, Taneja H. The small, disloyal fake news audience: The role of audience availability in fake news consumption[J]. New media \& society, 2018, 20(10): 3720-3737.

[18] Clements K W, Si J. Engel's law, diet diversity, and the quality of food consumption[J]. American Journal of Agricultural Economics, 2018, 100(1): 1-22.

[19] Bergström A, JervelyckeBelfrage M. News in social media: Incidental consumption and the role of opinion leaders[J]. Digital Journalism, 2018, 6(5): 583-598.

[20] Nguyen H V, Nguyen C H, Hoang T T B. Green consumption: Closing the intention - behavior gap[J]. Sustainable Development, 2019, 27(1): 118-129.

[21] McDowall W, Geng Y, Huang B, et al. Circular economy policies in China and Europe[J]. Journal of Industrial Ecology, 2017, 21(3): 651-661.

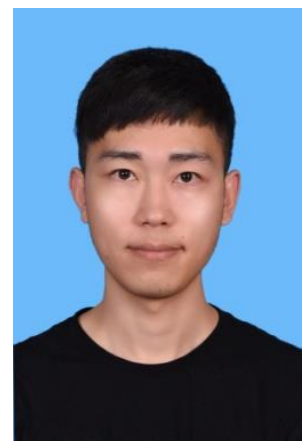

Longyue Bai is a $\mathrm{PhD}$ candidate working on journalism and communication in the College of the Humanities in Jilin University, China, His research interests include consumer culture and advertising culture, 2 papers published. 